$\mathrm{J}$ o u r n a $\mathrm{l}$ of

Mathematics

and Applications

JMA No 36, pp 35-62 (2013)

\title{
On duality between order and algebraic structures in Boolean systems
}

\author{
Aneta Dadej and Katarzyna Halik
}

\author{
Submitted by: Andrzej Kamiński
}

\begin{abstract}
We present an extension of the known one-to-one correspondence between Boolean algebras and Boolean rings with unit being two types of Boolean systems endowed with order and algebraic structures, respectively. Two equivalent generalizations of Boolean algebras are discussed. We show that there is a one-to-one correspondence between any of the two mentioned generalized Boolean algebras and Boolean rings without unit.
\end{abstract}

AMS Subject Classification: 06E05, 06E20, 06E75

Keywords and Phrases: lattice, 0-lattice, join-semilattice, 0-join-semilattice, distributive 0-lattice, B-ring, $\Delta$-join-semilattices, Boolean algebra, algebraic ring, Boolean ring

\section{Introduction}

There exists a beautiful one-to-one correspondence between the following two notions defined in two distinct ways: (i) a Boolean algebra, introduced in terms of a partially ordered set (a poset for short) as a 0-1-lattice which is distributive and complemented; (ii) a Boolean ring with unit, i.e. an algebraic ring with idempotent multiplication which contains a unit. The correspondence between the Boolean systems (i) and (ii) can be described by suitable homomorphisms which transmit the order structure of posets to the algebraic structure of Boolean rings and vice versa, so one can say about some kind of duality between the two structures (cf. Introduction in [7]). This fact is well known and presented in numerous papers and monographs (see e.g. [41], [42], [43], [3], [40], [45], [44], [24], [2], [21]).

A similar duality holds in a more general but still classical situation, when both (i) and (ii) above are considered without units, roughly speaking. More exactly, in terms of the notions used in this paper, there is a bijection between (I) B-rings, a kind of generalized Boolean algebras, lattices in a given poset $X$, in which $\sup X$ may not

COPYRIGHT (C) by Publishing Department Rzeszów University of Technology P.O. Box 85, 35-959 Rzeszów, Poland 
exist (see Definition 6), and (II) Boolean rings, i.e. algebraic rings with idempotent multiplication in which a unit may not exist (see Definition 16).

Various types of further extensions of Boolean algebras and rings were investigated by many authors (see e.g. [23], [4], [10], [11], [5], [12], [6], [13], [14], [15], [7]). In particular, very interesting general duality results concerning generalized orthomodular lattices are given in [10] and [7].

In this paper, we discuss certain aspects of duality between order and algebraic structures of Boolean systems. To present mutual relations between $B$-rings and Boolean rings (Theorems 4 and 6 in section 5 ) we give a list of properties of the binary operation (denoted here by -) of difference in $B$-rings, defined as an extension of the partial binary operation $\ominus$ of proper difference. The latter is introduced under condition $(R)$ postulated for $B$-rings in distributive 0-lattice (see Definitions 6 and 7 ). Some properties of difference in $B$-rings collected in section 3 were partly formulated and proved in our earlier paper [9]. We recall them in Proposition 12 and 14, changing slightly the notation and completing the omitted proofs.

On the other hand, we present also another approach in which the starting point is not a 0-lattice (join and meet of any two elements exist), but a special type of 0 -join-semilattice (only join of any two elements exists), namely a $\Delta$-join-semilattice (see Definitions 2 and 13) which satisfy, in addition, conditions $\left(I_{m}\right)$ and $(\Delta)$. The conditions allow one to define uniquely the binary operation $\backslash$ of difference in $\Delta$-joinsemilattices and guarantee that meets of any two of its elements exist. Moreover, the distributivity law and condition $(R)$, assumed for $B$-rings, are satisfied. Consequently, $\Delta$-join-semilattices appear to be an equivalent description of $B$-rings (see Theorem 3 in section 5). The mutual relations between $\Delta$-join-semilattices and Boolean rings (Theorems 5 and 7 in section 5) are consequences of Theorems 4 and 6 , in view of Theorem 3.

In our approach to Boolean systems based on the partial order structure a special role is played by various types of binary operations (partial and full) of difference. In this context it should be noted that there exist much more general, categorical and fuzzy ( $M V$-algebras) approaches, in which the notion of $D$-posets, introduced by $\mathrm{F}$. Kôpka and F. Chovanec (see [30], [32], [8], [31]) on the base of the partial operation of difference, plays an important role as a model in quantum probability theory. The notion was intensively investigated by R. Frič and M. Papčo (see [16]-[20], [33]-[35]).

Our interest in generalizing the notion of Boolean algebras to $B$-rings or $\Delta$-joinsemilattices is connected with the results of B. J. Pettis (see [36]) who extended to Boolean $\sigma$-rings certain theorems of W. Sierpiński (see [39]) on generated families of subsets of a given set (see also [28] and [22]). On the other hand, $B$-rings are natural objects for generalizing the theory of A. Rényi of conditional probability spaces (see [37], [38], [25], [26], [27], [1], [29]). We are going to discuss these issues in our forthcoming publications.

For completeness of the presentation we give full proofs of most of the results formulated in this paper. 


\section{Semilattices and lattices}

We start from certain basic definitions and their consequences. In particular, we recall some of definitions and results from our article [9].

All material in this section is given without proofs, because the properties formulated here either follow immediately from the respective definitions or can be received in a similar way as in [9].

Let $(X, \leq)$ be a poset, i.e. a partially ordered set, and let $A$ be its non-empty subset. We say that $\inf A[\sup A]$ exists in $X$ if there is an element $x \in X$ such that $1^{\circ} \quad x \leq y$ for all $y \in A$ and $2^{\circ} \quad X \ni x_{1} \leq y$ for all $y \in A$ implies $x_{1} \leq x\left[1^{\circ}\right.$ $y \leq x$ for all $y \in A$ and $2^{\circ} y \leq x_{1} \in X$ for all $y \in A$ implies $x \leq x_{1}$ ]. Clearly, $x$ satisfying $1^{\circ}$ and $2^{\circ}$ is unique and we denote $x:=\inf A[x:=\sup A]$. In particular, $\inf \{x\}=\sup \{x\}=x$ for each $x \in X$. If for given elements $x$ and $y$ of a poset $(X, \leq)$ the symbol $\inf \{x, y\}$ is used in any equality that we state is true, it means that $\inf \{x, y\}$ exists in $X$ (and the equality holds).

Definition 1. A poset $(X, \leq)$ is called a join-semilattice if

$$
\forall_{x, y \in X} \quad \sup \{x, y\} \text { exists in } X \text {. }
$$

The element $\sup \{x, y\}$ is called the join of $x$ and $y$ (see [3], p. 6, Definition).

If a given poset $(X, \leq)$ is a join-semilattice we denote

$$
x \vee y:=\sup \{x, y\}
$$

for any $x, y \in X$. Formula (2.1) determines the binary operation $\vee$ of join on $X$. We denote further a given join-semilattice $(X, \leq)$ by $(X, \vee)$ to mark explicitly the operation $\vee$ of join defined by (2.1). Let us remark that $\inf \{x, y\}$ for given elements $x$ and $y$ of a join-semilattice $(X, \vee)$, called the meet of $x$ and $y$ (see [3], p. 6, Definition), may exist or not, so the corresponding operation of meet may be treated, in general, as a partial binary operation on $X$.

In an analogous way we can define a meet-semilattice with a similar remark. We will not discuss further the notion of meet-semilattice which is dual to join-semilattice.

In Propositions 1 and 2 below we formulate a list of properties of join-semilattices corresponding to the respective properties of lattices given in [9] (see Statement 1), denoting them by $(j)$ with the corresponding indices.

Proposition 1. Let $(X, \vee)$ be a join-semilattice. Then

$$
\sup \left\{x_{1}, \ldots, x_{n}\right\} \quad \text { exists in } X
$$

for arbitrary $n \in \mathbb{N}$ and $x_{1}, \ldots, x_{n} \in X$. Moreover,

$$
\sup \left\{x_{1}, \ldots, x_{n}\right\}=\sup \left\{\sup \left\{x_{1}, \ldots, x_{n-1}\right\}, x_{n}\right\}
$$

for each $n \in \mathbb{N} \backslash\{1\}$ and $x_{1}, \ldots, x_{n}$ in $X$. 
Proposition 2. Let $(X, \vee)$ be a join-semilattice. Then

$\left(j_{2}\right) \quad x \vee x=x$,

$\left(j_{3}\right) \quad x \vee y=y \vee x$,

$\left(j_{5}\right) \quad(x \vee y) \vee z=x \vee(y \vee z)$,

(j6) $\quad x \leq x \vee y, \quad y \leq x \vee y$

$\left(j_{7}\right) \quad x \leq y \Leftrightarrow x \vee y=y$

$\left(j_{8}\right) \quad x \leq y \Rightarrow z \vee x \leq z \vee y$

for arbitrary $x, y, z \in X$.

Definition 2. A join-semilattice $(X, \vee)$ is called $(a)$ 0-join-semilattice; (b) 1-joinsemilattice; (c) 0-1-join-semilattice, whenever

$(J 0) \quad \inf X \quad$ exists in $X$;

(J1) $\quad \sup X$ exists in $X$;

$(J 2) \quad \inf X$ and $\sup X$ exist in $X$,

respectively.

The elements $\inf X$ and $\sup X$ are called the zero and the unit (more exactly: the order zero and the order unit) in a given join-semilattice $(X, \vee)$ and denoted by 0 and 1 , respectively. We denote a given $(a)$ 0-join-semilattice; $(b)$ 1-join-semilattice; $(c)$ 0 -1-join-semilattice $(X, \vee)$ by $(a)(X, \vee, 0) ;(b)(X, \vee, 1) ;(c)(X, \vee, 0,1)$, respectively, to mark explicitly the existence of the zero or/and the unit in the join-semilattice $(X, \vee)$.

Proposition 3. If $(X, \vee, 0)$ is a 0 -join-semilattice, then

$$
0 \leq x, \quad x \vee 0=0 \vee x=x, \quad \inf \{x, 0\}=0
$$

for each $x \in X$. If $(X, \vee, 1)$ is a 1-join-semilattice, then

$$
x \leq 1, \quad x \vee 1=1 \vee x=1, \quad \inf \{x, 1\}=x
$$

for each $x \in X$.

In the sequel, we will not discuss the notions of 1 -join-semilattice or $0-1$-joinsemilattice.

Definition 3. A poset $(X, \leq)$ is called a lattice if

$$
\forall_{x, y \in X} \quad \inf \{x, y\} \text { and } \sup \{x, y\} \text { exist in } X \text {. }
$$

In other words, a poset $(X, \leq)$ is a lattice if it is a join-semilattice and a meetsemilattice. 
If a given poset $(X, \leq)$ is a lattice we denote

$$
x \wedge y:=\inf \{x, y\} \quad \text { and } \quad x \vee y:=\sup \{x, y\}
$$

for any $x, y \in X$. The formulae in (2.2) define the binary operations $\wedge$ and $\vee$ on $X$, respectively. Any lattice $(X, \leq)$ with the operations $\wedge$ of meet and $\vee$ of join given by (2.2) will be denoted by $(X, \wedge, \vee)$.

Next, we recall some properties of an arbitrary lattice (see [9]).

Proposition 4. Let $(X, \wedge, \vee)$ be a lattice. Then

$$
\inf \left\{x_{1}, \ldots, x_{n}\right\} \text { and } \sup \left\{x_{1}, \ldots, x_{n}\right\} \text { exist in } X
$$

for arbitrary $n \in \mathbb{N}$ and $x_{1}, \ldots, x_{n} \in X$. Moreover,

$$
\inf \left\{x_{1}, \ldots, x_{n}\right\}=\inf \left\{\inf \left\{x_{1}, \ldots, x_{n-1}\right\}, x_{n}\right\}
$$

and

$$
\sup \left\{x_{1}, \ldots, x_{n}\right\}=\sup \left\{\sup \left\{x_{1}, \ldots, x_{n-1}\right\}, x_{n}\right\}
$$

for each $n \in \mathbb{N} \backslash\{1\}$ and $x_{1}, \ldots, x_{n}$ in $X$.

Proposition 5. Let $(X, \wedge, \vee)$ be a lattice. Then

$\left(l_{2}\right) \quad x \wedge x=x, \quad x \vee x=x$,

$\left(l_{3}\right) \quad x \wedge y=y \wedge x, \quad x \vee y=y \vee x$,

$\left(l_{4}\right) \quad(x \wedge y) \wedge z=x \wedge(y \wedge z)$,

$\left(l_{5}\right) \quad(x \vee y) \vee z=x \vee(y \vee z)$,

$\left(l_{6}\right) \quad x \wedge y \leq x \leq x \vee y, \quad x \wedge y \leq y \leq x \vee y$,

$\left(l_{7}\right) \quad x \leq y \Leftrightarrow x \wedge y=x \Leftrightarrow x \vee y=y$,

$\left(l_{8}\right) \quad x \leq y \Rightarrow z \wedge x \leq z \wedge y \quad \& \quad z \vee x \leq z \vee y$,

$\left(l_{9}\right) \quad(x \wedge z) \vee(y \wedge z) \leq(x \vee y) \wedge z$,

$\left(l_{10}\right) \quad(x \wedge y) \vee z \leq(x \vee z) \wedge(y \vee z)$

for arbitrary $x, y, z \in X$.

Definition 4. A lattice $(X, \wedge, \vee)$ is called distributive if

$$
(x \vee y) \wedge z=(x \wedge z) \vee(y \wedge z), \quad x, y, z \in X,
$$

or

$$
(x \wedge y) \vee z=(x \vee z) \wedge(y \vee z), \quad x, y, z \in X
$$


Remark 1. Conditions $(D)$ and $\left(D^{\prime}\right)$ are equivalent (see e.g. [3], p. 11, Theorem $9)$.

Definition 5. A lattice $(X, \wedge, \vee)$ is called (a) 0-lattice; (b) 1-lattice; (c) 0-1-lattice, whenever

$(L 0) \quad \inf X \quad$ exists in $X$;

(L1) $\sup X$ exists in $X$;

(L2) $\quad \inf X$ and $\sup X$ exist in $X$,

respectively.

The elements $\inf X$ and $\sup X$ are called the zero and the unit (more exactly: the order zero and the order unit) in a given lattice $(X, \wedge, \vee)$ and denoted by 0 and 1 , respectively. We denote a given $(a)$ 0-lattice; $(b)$ 1-lattice; $(c) 0$-1-lattice $(X, \wedge, \vee)$ by (a) $(X, \wedge, \vee, 0),(b)(X, \wedge, \vee, 1),(c)(X, \wedge, \vee, 0,1)$, respectively, to mark explicitly the existence of the zero or/and the unit in the lattice $(X, \wedge, \vee)$.

Proposition 6. If $(X, \wedge, \vee, 0)$ is a 0 -lattice, then

$$
0 \leq x, \quad x \vee 0=0 \vee x=x, \quad x \wedge 0=0 \wedge x=0
$$

for any $x \in X$. If $(X, \wedge, \vee, 1)$ is a 1-lattice, then

$$
x \leq 1, \quad x \vee 1=1 \vee x=1, \quad x \wedge 1=1 \wedge x=x
$$

for any $x \in X$.

\section{Properties of $B$-rings}

Definition 6. A distributive 0-lattice $(X, \wedge, \vee, 0)$ is called a $B$-ring if it satisfies the following condition:

$$
\forall_{x, y \in X, y \leq x} \exists_{z \in X} \quad z \wedge y=0 \quad \text { and } \quad z \vee y=x,
$$

i.e. if the poset $(X, \leq)$ satisfies conditions $(L),(L 0),(D)$ and $(R)$.

Definition 7. Let $(X, \wedge, \vee, 0)$ be a $B$-ring and $x, y$ be elements of $X$ such that $y \leq x$. Then by a proper difference of $x$ and $y$ we mean an element $z$ of $X$ satisfying the identities in $(R)$.

Let us recall the following known uniqueness result (see e.g. [3], p. 12, Theorem 10):

Theorem 1. Let $(X, \wedge, \vee)$ be a distributive lattice and let $y, z_{1}, z_{2}$ be its arbitrary elements. If $z_{1} \wedge y=z_{2} \wedge y$ and $z_{1} \vee y=z_{2} \vee y$, then $z_{1}=z_{2}$. 
Remark 2. In any distributive 0-lattice $(X, \wedge, \vee, 0)$, if for given $x, y \in X$ with $y \leq x$ there exists an element $z \in X$ satisfying the two equalities in $(R)$, then this element, the proper difference of $x$ and $y$, is unique, in view of Theorem 1 .

For any elements $x$ and $y$ such that $y \leq x$ of any distributive 0-lattice, we denote the proper difference of $x$ and $y$ by $x \ominus y$, i.e.

$$
x \ominus y=z \quad(y \leq x) \quad \text { if } \quad y \wedge z=0 \quad \text { and } \quad y \vee z=x .
$$

In an arbitrary $B$-ring $(X, \wedge, \vee, 0)$, formula (3.3) determines the partial binary operation $\ominus$ of proper difference in $X$ defined for all pairs of elements $x, y \in X$ such that $y \leq x$. Though the operation $\ominus$ of proper difference does not formally appear in Definition 6 it is uniquely determined, due to condition $(R)$ and Theorem 1, via formula (3.3). Therefore we will use in the sequel the notation $(X, \wedge, \vee, \ominus, 0)$ for $B$-rings, i.e. for those distributive 0-lattices $(X, \wedge, \vee, 0)$ which satisfy condition $(R)$.

We formulate below some properties of the operation $\ominus$. We start with the assertions, which are direct consequences of $(R),\left(l_{6}\right)$ and the above notation.

Proposition 7. If $(X, \wedge, \vee, \ominus, 0)$ is a B-ring, then

$$
\forall_{x, y \in X}, y \leq x \quad x \ominus y \leq x, \quad(x \ominus y) \wedge y=0, \quad(x \ominus y) \vee y=x .
$$

Proposition 8. If $(X, \wedge, \vee, \ominus, 0)$ is a B-ring, then

$$
\forall x, y \in X, y \leq x \quad x \ominus(x \ominus y)=y .
$$

The following de Morgan properties of the operation $\ominus$ are true:

Proposition 9. If $(X, \wedge, \vee, \ominus, 0)$ is a B-ring, then

$$
a \ominus(x \wedge y)=(a \ominus x) \vee(a \ominus y) \quad \text { and } \quad a \ominus(x \vee y)=(a \ominus x) \wedge(a \ominus y)
$$

for arbitrary $a, x, y \in X$ such that $x \leq a$ and $y \leq a$.

Proof. Fix $a, x, y \in X$ with $x \leq a$ and $y \leq a$. Taking into account Proposition 7, we can collect the relations:

$$
x \leq a, \quad y \leq a, \quad a \ominus x \leq a, \quad a \ominus y \leq a .
$$

Moreover, according to $(R)$ and Remark 2, we have

$$
x \wedge(a \ominus x)=y \wedge(a \ominus y)=0 \quad \text { and } \quad x \vee(a \ominus x)=y \vee(a \ominus y)=a .
$$

Applying $(D),\left(l_{6}\right),\left(l_{8}\right)$ and the first part of $(3.5)$, we get

$$
(x \wedge y) \wedge[(a \ominus x) \vee(a \ominus y)] \leq[x \wedge(a \ominus x)] \vee[y \wedge(a \ominus y)]=0
$$

and, consequently,

$$
(x \wedge y) \wedge[(a \ominus x) \vee(a \ominus y)]=0 .
$$


On the other hand, taking into account (3.4), using properties $\left(l_{2}\right),\left(l_{3}\right),\left(l_{5}\right)-\left(l_{8}\right)$, the distributivity in the form of $\left(D^{\prime}\right)$ and the second part of (3.5), we obtain the following chain of equalities:

$$
\begin{aligned}
(x & \wedge y) \vee[(a \ominus x) \vee(a \ominus y)] \\
& =[(x \wedge y) \vee(a \ominus x)] \vee[(x \wedge y) \vee(a \ominus y)] \\
& =[(x \vee(a \ominus x)) \wedge(y \vee(a \ominus x))] \vee[(x \vee(a \ominus y)) \wedge(y \vee(a \ominus y))] \\
& =[a \wedge(y \vee(a \ominus x))] \vee[(x \vee(a \ominus y)) \wedge a] \\
& =[y \vee(a \ominus x)] \vee[x \vee(a \ominus y)] \\
& =[x \vee(a \ominus x)] \vee[y \vee(a \ominus y)],
\end{aligned}
$$

i.e., applying again the second part of $(3.5)$ and $\left(l_{2}\right)$, we get

$$
(x \wedge y) \vee[(a \ominus x) \vee(a \ominus y)]=a .
$$

According to condition $(R)$ and Remark 2, the equalities (3.6) and (3.7) mean that the first identity in Proposition 9 holds.

The second identity can be shown in an analogous way.

Definition 8. A $B$-ring $(X, \wedge, \vee, \ominus, 0)$ is called a $B$-algebra if $\sup X$ exists in $X$, i.e. if conditions $(L),(L 2),(D)$ and $(R)$ are satisfied.

We denote $\sup X$ in a $B$-algebra by 1 and, consequently, the given $B$-algebra by $(X, \wedge, \vee, \ominus, 0,1)$.

The following assertion is straightforward:

Proposition 10. If $(X, \wedge, \vee, \ominus, 0)$ is a B-ring and $a$ is a fixed element of $X$, then $\left(X_{a}, \wedge, \vee, \ominus, 0\right)$, where $X_{a}:=\{x \in X: x \leq a\}$, is a B-algebra with the unit $1_{a}:=\sup X_{a}=a$.

In an arbitrary $B$-algebra $(X, \wedge, \vee, \ominus, 0,1)$, for every $x \in X$, the following condition is satisfied:

$$
\left(C_{x}\right) \quad \exists_{z \in X} \quad z \wedge x=0 \quad \text { and } \quad z \vee x=1 .
$$

The condition results directly from $(R)$. An element $z$ satisfying the equalities in condition $\left(C_{x}\right)$ is unique for any $x \in X$ (see Remark 2).

Definition 9. Let $(X, \wedge, \vee, 0,1)$ be a 0-1-lattice. Fix $x \in X$. By a complement of $x$ we mean an element $z \in X$ satisfying condition $\left(C_{x}\right)$. If condition $\left(C_{x}\right)$ is satisfied for every $x \in X$, then the 0 -1-lattice $(X, \wedge, \vee, 0,1)$ is called complemented (see [3], p. $16)$.

Remark 3. In any distributive 0 -1-lattice $(X, \wedge, \vee, 0,1)$, if for a given $x \in X$ condition $\left(C_{x}\right)$ is satisfied by $z \in X$, then this $z$, the complement of $x$, is unique, in view of Theorem 1 . 
In a distributive 0 -1-lattice $(X, \wedge, \vee, 0,1)$, we denote, for any element $x \in X$ for which condition $\left(C_{x}\right)$ is satisfied, the complement of $x$ by $x^{\prime}$. In particular, if a given distributive 0 -1-lattice $(X, \wedge, \vee, 0,1)$ is complemented we may consider the unary operation ' of complement in $X$, denoting such a lattice by $\left(X, \wedge, \vee,{ }^{\prime}, 0,1\right)$.

Definition 10. According to [3], pp. 17-18, by a Boolean algebra we mean a complemented distributive 0-1-lattice $\left(X, \wedge, \vee,{ }^{\prime}, 0,1\right)$ such that the unary operation ' of complement satisfies the following conditions:

(A1) $\quad x \wedge x^{\prime}=0, \quad x \vee x^{\prime}=1$,

(A2) $\quad\left(x^{\prime}\right)^{\prime}=x$

(A3) $\quad(x \wedge y)^{\prime}=x^{\prime} \vee y^{\prime}, \quad(x \vee y)^{\prime}=x^{\prime} \wedge y^{\prime}$ for any $x, y \in X$.

Theorem 2. Every $B$-algebra $(X, \wedge, \vee, \ominus, 0,1)$ is a Boolean algebra $\left(X, \wedge, \vee,{ }^{\prime}, 0,1\right)$ with the unary operation' defined by

$$
x^{\prime}:=1 \ominus x
$$

for any $x \in X$. Conversely, every Boolean algebra $\left(X, \wedge, \vee,{ }^{\prime}, 0,1\right)$ is a B-algebra $(X, \wedge, \vee, \ominus, 0,1)$ with the binary operation $\ominus$ defined by

$$
x \ominus y:=x \wedge y^{\prime}
$$

for $x, y \in X$ such that $y \leq x$.

Proof. Let $(X, \wedge, \vee, \ominus, 0,1)$ be a $B$-algebra. Hence, in particular, the 0-1-lattice $(X, \wedge, \vee, 0,1)$ is distributive and complemented with the operation ' of complement defined by formula (3.8). That the operation ' satisfies conditions $(A 1)$ and $(A 2)$ follows from Propositions 7 and 8 , respectively, applied for $x:=1$ and $y:=x$. That condition (A3) is satisfied follows from Proposition 9 for $a:=1$. This proves the first part of Theorem 2 .

To prove the second part, since a given Boolean algebra $\left(X, \wedge, \vee,{ }^{\prime}, 0,1\right)$ is a distributive 0 -1-lattice, it suffices to verify that condition $(R)$ is fulfilled. Fix $x, y \in X$ such that $y \leq x$ and put $z:=x \wedge y^{\prime}$. We obtain

$$
y \wedge z=y \wedge\left(x \wedge y^{\prime}\right)=\left(y \wedge y^{\prime}\right) \wedge x=0
$$

and

$$
y \vee z=y \vee\left(x \wedge y^{\prime}\right)=(y \vee x) \wedge\left(y \vee y^{\prime}\right)=x,
$$

applying the two equalities in $(A 1)$ as well as properties $\left(l_{3}\right),\left(l_{4}\right),\left(l_{7}\right),(l 0),(l 1)$ and $\left(D^{\prime}\right)$. Hence $z$ satisfies the equalities in $(R)$ and, by Theorem 1 and formula (3.3), $z=x \ominus y$. This completes the proof of Theorem 2 .

In any $B$-ring $(X, \wedge, \vee, \ominus, 0)$, the partial binary operation $\ominus$ can be extended to the binary operation (denoted here by - ) on $X$ defined for an arbitrary pair of elements of $X$ in the following way: 
Definition 11. If $(X, \wedge, \vee, \ominus, 0)$ is a $B$-ring, then we define the difference $x-y$ of $x$ and $y$ by

$$
x-y:=x \ominus(x \wedge y)
$$

for arbitrary $x, y \in X$.

Notice that definition in (3.9) makes sense, because $x \wedge y \leq x$ for any $x, y \in X$, due to property $\left(l_{6}\right)$. The binary operation - of difference defined above is consistent with the partial binary operation $\ominus$ of proper difference, introduced in Definition 7, according to the following obvious property:

Proposition 11. Let $(X, \wedge, \vee, \ominus, 0)$ be a B-ring. If $x, y \in X$ and $y \leq x$, then $x-y=x \ominus y$.

It should be noted that the above extension - of the operation $\ominus$ of proper difference satisfies an extended form of condition $(R)$ with the corresponding uniqueness property (cf. Theorem 1 and Remark 2). Namely, the following assertion is true:

Proposition 12. Let $(X, \wedge, \vee, \ominus, 0)$ be a B-ring. Then

$$
\forall_{x, y \in X} \exists_{z \in X} \quad z \wedge y=0 \quad \text { and } \quad z \vee y=x \vee y,
$$

and an element $z$ in $(\bar{R})$ is unique, given by $z:=x-y$.

Proposition 12 is a consequence of the following two facts. That for arbitrary $x, y \in X$ in a $B$-ring the two identities required in condition $(\bar{R})$ are satisfied by $z:=x-y$ follows from the two last equalities from part $2^{\circ}$ of Proposition 14 proved below. That this $z$ is unique follows directly from the known uniqueness result cited above as Theorem 1 .

In addition to the uniqueness property concerning the operation of difference, formulated in Proposition 12, we will give below a series of properties of this operation in any $B$-ring. Let us start with the following easy consequence of Definition 11 and Proposition 12:

Proposition 13. Let $(X, \wedge, \vee, \ominus, 0)$ be a B-ring. We have

$$
x-0=x \ominus 0=x, \quad x-x=x \ominus x=0, \quad 0-x=0
$$

for every $x \in X$.

Properties 1-15 formulated in [9] contain the statement expressed in Proposition 12 and several other properties recalled in Proposition 14 below. Proofs of some of the properties were omitted in [9]. We complete below all the omitted proofs. The properties will be used in the proofs of certain characterizations given in section .

Proposition 14. Let $(X, \wedge, \vee, \ominus, 0)$ be a B-ring. The following properties of the binary operation - of difference on $X$ hold true:

$$
1^{\circ} \quad x-y \leq x, \quad(x-y) \wedge(x \wedge y)=0, \quad(x-y) \vee(x \wedge y)=x,
$$




$$
\begin{array}{ll}
2^{\circ} & (x-y) \wedge(y-x)=0, \quad(x-y) \wedge y=0, \quad(x-y) \vee y=x \vee y, \\
3^{\circ} & (x-y) \vee(y-x) \vee(x \wedge y)=x \vee y, \\
4^{\circ} & x \leq y \Rightarrow(z-y) \wedge x=0, \quad x \leq y \Rightarrow(z-y)-x=z-y, \\
5^{\circ} & x \leq y \Rightarrow z-y \leq z-x, \\
6^{\circ} & z-(x \vee y)=(z-x) \wedge(z-y), \quad z-(x \wedge y)=(z-x) \vee(z-y), \\
7^{\circ} & z-(x \vee y)=(z-x)-y, \quad(x \vee y)-z=(x-z) \vee(y-z), \\
8^{\circ} & (x-y) \wedge z=(x \wedge z)-(y \wedge z)
\end{array}
$$

for any $x, y, z \in X$.

Proof. Since $x-y=x \ominus(x \wedge y)$, by the definition in (3.9), the three properties in $1^{\circ}$ follow directly from Proposition 7 .

To prove the first property in $2^{\circ}$ assume that

$$
u \leq x-y=x \ominus(x \wedge y) \quad \text { and } \quad u \leq y-x=y \ominus(x \wedge y) .
$$

Therefore, by the first property in $1^{\circ}$, we have $u \leq x, u \leq y$ and, consequently, $u \leq x \wedge y$, due to $\left(l_{8}\right),\left(l_{3}\right.$ and $\left(l_{2}\right)$. Hence $u=0$, in view of (3.11) and the second assertion in Proposition 7. Thus

$$
(x-y) \wedge(y-x)=\inf \{x-y, y-x\}=0,
$$

as required.

To show the two remaining properties in $2^{\circ}$ together with property $3^{\circ}$ we use the representations:

$$
y=[y \ominus(x \wedge y)] \vee(x \wedge y)=(y-x) \vee(x \wedge y),
$$

following from Proposition 7 and (3.9). Applying (3.13), the distributivity condition $(D)$ as well as the properties $\left(l_{2}\right),\left(l_{3}\right)$ and $\left(l_{5}\right)$, we obtain

$$
\begin{aligned}
(x-y) \wedge y & =(x-y) \wedge[(y-x) \vee(x \wedge y)] \\
& =[(x-y) \wedge(y-x)] \vee[(x-y) \wedge(x \wedge y)]=0,
\end{aligned}
$$

and

$$
\begin{aligned}
(x-y) \vee y & =(x-y) \vee(y-x) \vee(x \wedge y) \\
& =[(x-y) \vee(x \wedge y)] \vee[(y-x) \vee(y \wedge x)]=x \vee y,
\end{aligned}
$$

in view of (3.12) and the two last identities in $1^{\circ}$. The proof of the third property in $2^{\circ}$ was given also in [9] (see the proof of Property 5 there).

To prove the assertions in $4^{\circ}$ and $5^{\circ}$ assume that $x, y, z \in X$ and $x \leq y$. We have

$$
(z-y) \wedge x \leq(z-y) \wedge y=0,
$$


by $\left(l_{8}\right)$ and the second identity in $2^{\circ}$, and hence

$$
z-y=[(z-y)-x] \vee[(z-y) \wedge x]=(z-y)-x,
$$

by the third identity in $1^{\circ}$. The assertions in $4^{\circ}$ follow from (3.14) and (3.15).

Further, $z \wedge x \leq z \wedge y \leq z$ and $z-y \leq z$, according to our assumption and due to $\left(l_{6}\right),\left(l_{8}\right)$ and the first property in $1^{\circ}$, so we may write

$$
z-y=(z-y) \wedge z=[z \ominus(z \wedge y)] \wedge[(z \ominus(z \wedge x)) \vee(z \wedge x)],
$$

in view of $\left(l_{7}\right)$ and Proposition 7. But, since $z \wedge x \leq z \wedge y$, we have

$$
[z \ominus(z \wedge y)] \wedge(z \wedge x)=[z-(z \wedge y)] \wedge(z \wedge x)=0
$$

by the first property in $4^{\circ}$, already proved. Therefore, in view of the distributivity condition $(D)$, the equations in (3.16) yield

$$
z-y=[z \ominus(z \wedge y)] \wedge[(z \ominus(z \wedge x))]=(z-y) \wedge(z-x),
$$

which means, due to $\left(l_{7}\right)$, that the assertion in $5^{\circ}$ is proved (see also the proof of Property 10 in [9]).

The two assertions in $6^{\circ}$ are consequences of Definition 11 and Proposition 9 . Namely, we conclude from them the identity

$$
\begin{aligned}
z-(x \vee y) & =z \ominus[z \wedge(x \vee y)]=z \ominus[(z \wedge x) \vee(z \wedge y)] \\
& =[z \ominus(z \wedge x)] \wedge[z \ominus(z \wedge y)]=(z-x) \wedge(z-y)
\end{aligned}
$$

using additionally the distributivity property $(D)$, and the identity

$$
\begin{aligned}
z-(x \wedge y) & =z \ominus[z \wedge(x \wedge y)]=z \ominus[(z \wedge x) \wedge(z \wedge y)] \\
& =[z \ominus(z \wedge x)] \vee[z \ominus(z \wedge y)]=(z-x) \vee(z-y)
\end{aligned}
$$

using here, in addition, properties $\left(l_{2}\right)-\left(l_{4}\right)$.

Complete proofs of the three remaining properties formulated in $7^{\circ}$ and $8^{\circ}$ are given in [9] (see Properties 13-15).

We will need also some additional properties of the operation of difference in $B$-rings.

Proposition 15. Let $(X, \wedge, \vee, \ominus, 0)$ be a B-ring. Then

$$
\begin{aligned}
& {[(x-y) \vee(y-x)] \wedge(x \wedge y)=0} \\
& (x-y) \wedge[z-(x \vee y)]=0, \quad(y-x) \wedge[z-(x \vee y)]=0, \\
& (x-y) \wedge(x \wedge y \wedge z)=0, \quad(y-x) \wedge(x \wedge y \wedge z)=0
\end{aligned}
$$

for any $x, y, z \in X$. 
Proof. Applying $(D),\left(l_{6}\right),\left(l_{8}\right)$ and the second property in part $2^{\circ}$ of Proposition 14 , we get

$$
[(x-y) \vee(y-x)] \wedge(x \wedge y) \leq[(x-y) \wedge y] \vee[(y-x) \wedge x]=0,
$$

i.e. equality (3.17) is shown.

In view of property $\left(l_{3}\right)$, it remains to prove the first equalities in (3.18) and (3.19). We have $x-y \leq x$ and $z-(x \vee y) \leq z-x$, by the first assertion in $1^{\circ}$ and by $5^{\circ}$ from Proposition 14. Therefore, using $\left(l_{6}\right)$ and $\left(l_{2}\right)$, we get

$$
(x-y) \wedge[z-(x \vee y)] \leq x \wedge(z-x)=0,
$$

and, similarly,

$$
(x-y) \wedge(x \wedge y \wedge z) \leq[x-(x \wedge y)] \wedge(x \wedge y)=0,
$$

due to the second assertion in part $2^{\circ}$ of Proposition 14. The required equalities are proved and the proof of the proposition is completed.

\section{Properties of $\Delta$-join-semilattices}

Definition 12. We say that a 0 -join-semilattice $(X, \vee, 0)$ is meet-invariant if the following implication holds:

$\left(I_{m}\right) \quad$ if $\inf \{x, y\}=0$, then $\inf \{x \vee z, y \vee z\}=z \quad$ for any $x, y, z \in X$.

Proposition 16. Each distributive 0-lattice $(X, \wedge, \vee, 0)$ is meet-invariant.

Proof. Suppose that $x, y \in X$ and $\inf \{x, y\}=0$. Then, from the distributivity law $\left(D^{\prime}\right)$, we have

$$
\inf \{x \vee z, y \vee z\}=(x \vee z) \wedge(y \vee z)=(x \wedge y) \vee z=z
$$

for every $z \in X$, i.e. condition $\left(I_{m}\right)$ is fulfilled.

Definition 13. We say that a 0 -join-semilattice $(X, \vee, 0)$ is a $\Delta$-join-semilattice if it is meet-invariant and satisfies the following condition:

$$
\forall_{x, y \in X} \exists_{z \in X} \quad \inf \{y, z\}=0 \quad \text { and } \quad y \vee z=x \vee y .
$$

In particular, condition $(\Delta)$ in any 0 -join-semilattice $(X, \vee, 0)$ yields:

$$
\left(\Delta_{0}\right) \quad \forall_{x, y \in X, y \leq x} \exists_{z \in X} \quad \inf \{y, z\}=0 \quad \text { and } \quad y \vee z=x .
$$

Proposition 17. If $(X, \vee, 0)$ is a $\Delta$-join-semilattice, then for every pair of elements $x, y \in X$ there exists a unique $z$ such that the equalities in condition $(\Delta)$ are satisfied. In particular, a similar uniqueness holds in case of $\left(\Delta_{0}\right)$. 
Proof. Fix $x, y \in X$. Suppose that there exist elements $z_{1}, z_{2}$ in $X$ satisfying condition $(\Delta)$, that is:

$$
\inf \left\{y, z_{i}\right\}=0 \quad \text { and } \quad y \vee z_{i}=x \vee y \quad(i=1,2) .
$$

Hence, by condition $\left(I_{m}\right)$ we have

$$
z_{1}=\inf \left\{y \vee z_{1}, z_{2} \vee z_{1}\right\}=\inf \left\{y \vee z_{2}, z_{1} \vee z_{2}\right\}=z_{2},
$$

which proves that the assertion is true.

Definition 14. Let $(X, \vee, 0)$ be a $\Delta$-join-semilattice and let $x, y$ be arbitrary elements of $X$. Then by a difference of $x$ and $y$ we mean an element $z$ of $X$ satisfying the identities in $(\Delta)$.

A difference of any elements $x$ and $y$ in an arbitrary $\Delta$-join-semilattice $(X, \vee, 0)$ exists and is unique (see condition $(\Delta)$ and Proposition 17). We denote the difference of elements $x$ and $y$ by $x \backslash y$, i.e.

$$
x \backslash y=z \quad \text { if } \quad \inf \{y, z\}=0 \quad \text { and } \quad y \vee z=x \vee y .
$$

If $(X, \vee, 0)$ is a $\Delta$-join-semilattice, then formula (4.20) determines the binary operation $\checkmark$ of difference in $X$. In particular, if $y \leq x$ we call $x \backslash y$ the proper difference of $x$ and $y$, i.e.

$$
x \backslash y=z \quad(y \leq x) \quad \text { if } \quad \inf \{y, z\}=0 \quad \text { and } \quad y \vee z=x .
$$

Remark 4. Though the operation $\backslash$ of difference does not formally appear in Definition 13 it is uniquely determined, due to conditions $(\Delta),\left(I_{m}\right)$ and Proposition 17 , via formula (4.20). Therefore we will use in the sequel the notation $(X, \vee, \backslash, 0)$ for $\Delta$-join-semilattices, i.e. for those 0 -join-semilattices $(X, \vee, 0)$ which are meet-invariant and satisfy condition $(\Delta)$.

Lemma 1. If $(X, \vee, \backslash, 0)$ is a $\Delta$-join-semilattice, then

$$
x \backslash y \leq x
$$

for any $x, y \in X$.

Proof. Let $z:=x \backslash y$. In view of Definition 14 the element $z$ satisfies condition $(\Delta)$, i.e. the following equalities hold:

$$
\inf \{y, z\}=0 \quad \text { and } \quad y \vee z=x \vee y .
$$

Then, by $\left(I_{m}\right)$ and (4.23), we have

$$
x=\inf \{y \vee x, z \vee x\}=\inf \{y \vee z, x \vee z\} .
$$

On the other hand, in view of $\left(j_{6}\right)$, we have the two relations:

$$
z \leq y \vee z \quad \text { and } \quad z \leq x \vee z
$$


which imply, due to (4.24), that

$$
z \leq \inf \{y \vee z, x \vee z\}=x .
$$

The relation $(4.22)$ is proved.

Lemma 2. If $(X, \vee, \backslash, 0)$ is a $\Delta$-join-semilattice, then $x \backslash(x \backslash y) \leq y$ for any $x, y \in X$.

Proof. Let $z:=x \backslash y$ and $u:=x \backslash(x \backslash y)=x \backslash z$. By Lemma 1 , we have $z \leq x$ and thus $x \backslash z$ is a proper difference. Hence, by Definition 14 (see (4.20) and (4.21)), we obtain the equalities

$$
\inf \{y, z\}=0 \quad \text { and } \quad y \vee z=x \vee y
$$

as well as the identities

$$
\inf \{z, u\}=0 \quad \text { and } \quad z \vee u=x .
$$

Then, in view of $(4.25),(4.26),\left(j_{3}\right)$ and $\left(j_{5}\right)$ we have

$$
y \vee z=x \vee y=(z \vee u) \vee y=(y \vee z) \vee u .
$$

Hence, by $\left(j_{7}\right)$, we get $u \leq y \vee z$. On the other hand, we have $u \leq u \vee y$, by $\left(j_{6}\right)$. The last two relations, the first equality in (4.26) and condition $\left(I_{m}\right)$ imply

$$
u \leq \inf \{z \vee y, u \vee y\}=y
$$

and the assertion is shown.

Lemma 3. Let $(X, \vee, \backslash, 0)$ be a $\Delta$-join-semilattice and $x, y, u \in X$. If $u \leq x \vee y$ and $\inf \{x, u\}=0$, then $u \leq y$.

Proof. Since $u \leq x \vee y$ and $u \leq u \vee y$, by $\left(j_{6}\right)$, we have

$$
u \leq \inf \{x \vee y, u \vee y\}=y
$$

in view of condition $\left(I_{m}\right)$ and the assumption that $\inf \{x, u\}=0$.

Lemma 4. Let $(X, \vee, \backslash, 0)$ be a $\Delta$-join-semilattice and $x, y, u \in X$. If $u \leq x$ and $u \leq y$, then $\inf \{u, x \backslash y\}=0$.

Proof. Let $z:=x \backslash y$. In view of Definition 14, the element $z$ satisfies condition $(\Delta)$, i.e. the following equalities hold:

$$
\inf \{y, z\}=0 \quad \text { and } \quad y \vee z=x \vee y .
$$

Let $u \leq x, u \leq y$ and let $v \leq u, v \leq x \backslash y=z$. Since $v \leq u \leq y$ and $v \leq z$, we have

$$
v \leq \inf \{y, z\}=0 \text {, }
$$

by (4.27). Hence $v=0$. Consequently, $\inf \{u, x \backslash y\}=0$. 
Lemma 5. Let $(X, \vee, \backslash, 0)$ be $a \Delta$-join-semilattice and $x, y, u \in X$. If $u \leq x$ and $u \leq y$, then $u \leq x \backslash(x \backslash y)$.

Proof. Let $y_{1}:=x \backslash y$ and $z_{1}:=x \backslash(x \backslash y)$. By Lemma 1 , we have $y_{1} \leq x$, so $x \backslash y_{1}$ is a proper difference. By Definition 14 (see (4.21)), we get

$$
\inf \left\{y_{1}, z_{1}\right\}=0 \quad \text { and } \quad y_{1} \vee z_{1}=x
$$

Since $u \leq x$, we have

$$
u \leq y_{1} \vee z_{1}
$$

due to the second equality in (4.28). Since $u \leq x$ and $u \leq y$, we have

$$
\inf \left\{u, y_{1}\right\}=\inf \{u, x \backslash y\}=0
$$

in view of Lemma 4. Hence, by Lemma 3, conditions (4.29) and (4.30) imply

$$
u \leq z_{1}=x \backslash(x \backslash y)
$$

Proposition 18. Let $(X, \vee, \backslash, 0)$ be a $\Delta$-join-semilattice. Then

$$
\inf \{x, y\}=x \backslash(x \backslash y) \in X,
$$

i.e. $\inf \{x, y\}$ exists in $X$ for any $x, y \in X$. Moreover, if $\wedge$ is a binary operation on $X$ given by

$$
x \wedge y:=\inf \{x, y\} \quad \text { for } x, y \in X,
$$

then $(X, \wedge, \vee, 0)$ is a 0 -lattice satisfying conditions $(R)$ and $\left(I_{m}\right)$.

Proof. Fix arbitrarily $x, y \in X$. In view of Lemma 1 and 2, we have

$$
x \backslash(x \backslash y) \leq x \quad \text { and } \quad x \backslash(x \backslash y) \leq y,
$$

so $x \backslash(x \backslash y)$ is a lower bound of elements $x, y$. Due to Lemma 5 , the element $x \backslash(x \backslash y)$ is the greatest lower bound of elements $x, y$, which means that the equality in (4.31) holds and so $\inf \{x, y\}$ exists in $X$.

Consequently, formula (4.32) well defines the binary operation $\wedge$ on $X$ and $(X, \wedge, \vee, 0)$ is a 0 -lattice which satisfies condition $(R)$. The latter results from the fact that $(R)$ is an equivalent form of condition $\left(\Delta_{0}\right)$ which is a particular case of condition $(\Delta)$, assumed for the given $\Delta$-join-semilattice $(X, \vee, \backslash, 0)$.

Remark 5. Notice that condition $\left(I_{m}\right)$ can be now expressed in the following equivalent form:

$\left(I_{m}^{\prime}\right) \quad(x \vee z) \wedge(y \vee z)=z$, whenever $x \wedge y=0 \quad$ for any $x, y, z \in X$. 
Proposition 19. Let $(X, \vee, \backslash, 0)$ be a $\Delta$-join-semilattice. Then the binary operation $\wedge$ given by (4.32) is distributive, i.e.

$$
(x \vee z) \wedge(y \vee z)=(x \wedge y) \vee z
$$

for arbitrary $x, y, z \in X$. Consequently $(X, \wedge, \vee, \ominus, 0)$ is a $B$-ring, where the partial binary operation $\ominus$ is given by

$$
x \ominus y:=x \backslash y
$$

for arbitrary $x, y \in X$ such that $y \leq x$.

Proof. Let $x, y, z$ be arbitrary elements of $X$ and denote

$$
u:=x \wedge y
$$

By (4.31) and (4.32) in Proposition 18, it follows that $u \in X$. Clearly, we have

$$
u \leq x, \quad u \leq y, \quad x \backslash u \leq x, \quad y \backslash u \leq y,
$$

where $x \backslash u$ and $y \backslash u$ are proper differences, by (4.34) and Lemma 1. Moreover,

$$
\inf \{u, x \backslash u\}=0=\inf \{u, y \backslash u\},
$$

by the first equality in $\left(\Delta_{0}\right)$.

To prove the identity

$$
\inf \{x \backslash u, y \backslash u\}=0
$$

suppose that $v \leq x \backslash u$ and $v \leq y \backslash u$. Hence, by the last two relations in (4.35), we have $v \leq x$ and $v \leq y$, so $v \leq \inf \{x, y\}=u$, in view of (4.34). But then (4.36) implies that $v=0$, which proves equality (4.37).

On the other hand, by the second equality in $\left(\Delta_{0}\right)$, we have

$$
x=u \vee(x \backslash u) \quad \text { and } \quad y=u \vee(y \backslash u) .
$$

Hence, by (4.38), (4.37) and $\left(I_{m}\right)$, we have

$$
\begin{aligned}
(x \vee z) \wedge(y \vee z) & =[(x \backslash u) \vee(u \vee z)] \wedge[(y \backslash u) \vee(u \vee z)] \\
& =u \vee z=(x \wedge y) \vee z,
\end{aligned}
$$

according to $\left(j_{3}\right),\left(j_{5}\right)$ and the notation in (4.34), i.e. the distributivity law (4.33) is proved.

\section{Duality theorems}

Theorem 3. Assume that $(X, \vee, \backslash, 0)$ is a $\Delta$-join-semilattice, where the binary operation $\backslash$ is given by (4.20), according to conditions $\left(I_{m}\right)$ and $(\Delta)$. Then $(X, \wedge, \vee, \ominus, 0)$ is a B-ring with the binary lattice operation $\wedge$, given by

$$
x \wedge y:=\inf \{x, y\}=x \backslash(x \backslash y) \quad \text { for } x, y \in X,
$$


and the partial binary operation $\ominus$ of proper difference, given by

$$
x \ominus y:=x \backslash y \quad \text { for } x, y \in X, y \leq x .
$$

Conversely, assume that $(X, \wedge, \vee, \ominus, 0)$ is a B-ring, where the partial binary operation $\ominus$ of proper difference is given by (3.3), according to condition $(R)$. Then $(X, \vee, \backslash, 0)$ is a $\Delta$-join-semilattice with the binary operation $\backslash$ given, according to $\left(I_{m}\right),(\Delta)$ and $(4.20)$, by

$$
x \backslash y:=x \ominus(x \wedge y) \quad \text { for } x, y \in X .
$$

Proof. That a given $\Delta$-join-semilattice $(X, \vee, \backslash, 0)$ is a $B$-ring follows directly from Propositions 18 and 19.

To show the second part of the assertion it suffices to notice that conditions $\left(I_{m}\right)$ and $(\Delta)$ in a given $B$-ring $(X, \wedge, \vee, \ominus, 0)$ follow from Propositions 16 and 12 , respectively, because the extension $(\bar{R})$ of condition $(R)$ is an equivalent form of condition $(\Delta)$.

The proof of Theorem 3 is completed.

Definition 15. By an algebraic ring $(X,+, \cdot)$ we mean a nonempty set $X$ endowed with two binary operations: + (addition) and - (multiplication) such that $(X,+)$ is an Abelian group and the multiplication is associative and distributive with respect to the addition. By a commutative ring we mean an algebraic ring $(X,+, \cdot)$ in which the operation $\cdot$ is commutative.

Definition 16. By a Boolean ring we mean an algebraic ring $(X,+, \cdot)$ in which the operation - of the multiplication is idempotent, i.e. $x^{2}=x$ for every $x \in X$. By a Boolean ring with unit we mean a Boolean ring in which there is a unique neutral element of the multiplication.

Remark 6. It follows from Lemma 8 below that every Boolean ring is commutative.

Let $(X, \wedge, \vee, \ominus, 0)$ be a $B$-ring. Define in $(X, \wedge, \vee, \ominus, 0)$ the two binary operations + and $\cdot$ as follows:

$$
x+y:=(x \vee y) \ominus(x \wedge y)=(x \vee y)-(x \wedge y)
$$

and

$$
x \cdot y:=x \wedge y .
$$

for arbitrary $x, y \in X$.

Remark 7. In any $B$-ring $(X, \wedge, \vee, \ominus, 0)$, the binary operation + on $X$ can be defined in two ways by formula (5.39), because $x \wedge y \leq x \vee y$ and thus the difference $u-v$ and the proper difference $u \ominus v$ of $u:=x \vee y$ and $v:=x \wedge y$ coincide, by Proposition 12 , for any $x, y \in X$. 
Due to our extension in Definition 11 of the partial binary operation $\ominus$ of proper difference to the binary operation - of difference in $B$-rings, the above definition of the operation + can be expressed in an equivalent form, given by formula (5.41) below. In fact, it follows from Proposition 20 proved below that formulae (5.39) and (5.41) stand for two equivalent definition of the binary operation + .

Formulae (5.39) and (5.41) correspond to the known representations of symmetric difference of two sets.

Proposition 20. Let $(X, \wedge, \vee, \ominus, 0)$ be a B-ring. Then

$$
x+y=(x-y) \vee(y-x)
$$

for arbitrary $x, y \in X$, where $x+y$ is given by formula (5.39).

Proof. Fix $x, y \in X$ and put $z:=(x-y) \vee(y-x)$. We have to prove that $z$ coincides with $x+y$, i.e. that $z=a \ominus b$, where $a:=x \vee y$ and $b:=x \wedge y$. According to Definition 7 and Remark 2 it suffices to verify that the respective equations in condition $(R)$ hold, i.e. we have the following identities: $z \wedge b=0$ and $z \vee b=a$. But they have been already proved in Proposition 15 in the form of (3.17) and in part $3^{\circ}$ of Proposition 14.

Now we will show some properties of a $B$-ring needed in the proof of Theorem 4 .

Lemma 6. Let $(X, \wedge, \vee, \ominus, 0)$ be a $B$-ring. Then

$$
z-(x+y)=[z-(x \vee z)] \vee(x \wedge y \wedge z)
$$

for arbitrary $x, y, z \in X$, where $x+y$ is given by formula (5.39).

Proof. We will use in the proof condition $(\bar{R})$, a generalization of condition $(R)$ proved in Proposition 12.

Fix $x, y, z \in X$ and denote $a:=x-y$ and $b:=y-x$, i.e.

$$
x+y=a \vee b,
$$

due to Proposition 20.

According to Proposition 12 it is enough to show the two equalities:

$$
(x+y) \wedge c=0 \quad \text { and } \quad(x+y) \vee c=(x+y) \vee z,
$$

where

$$
c:=[z-(x \vee y)] \vee(x \wedge y \wedge z) .
$$

Using definition (5.39) of $x+y$, we get

$$
(x+y) \wedge[z-(x \vee y)]=0,
$$

in view of the distributivity law $(D)$ as well as both equalities in (3.18), proved in Proposition 15. In a similar way the equalities in (3.19) imply that

$$
(x+y) \wedge(x \wedge y \wedge z)=0 .
$$


From (5.45) and (5.46) again using $(D)$, we obtain the first equality in (5.44).

To prove the second equality in (5.44), notice first that

$$
(x+y) \vee c \leq(x+y) \vee z,
$$

since $z-(x \vee y) \leq z$ and $x \wedge y \wedge z \leq z$, by part $1^{\circ}$ of Proposition 14 and $\left(l_{6}\right)$.

On the other hand, we have

$$
x \vee y=a \vee b \vee(x \wedge y), \quad a \wedge z \leq a, \quad b \wedge z \leq b,
$$

by property $3^{\circ}$ in Proposition 14 and $\left(l_{6}\right)$. In view of $(5.48)$ and $(D)$, we have

$$
\begin{aligned}
(x \vee y) \wedge z & \leq[(a \vee b) \vee(x \wedge y)] \wedge z \leq(a \vee b) \vee(x \wedge y \wedge z) \\
& =(x+y) \vee(x \wedge y \wedge z),
\end{aligned}
$$

according to $\left(l_{8}\right)$ and our notation (5.43). Hence, by the third identity in part $1^{\circ}$ in Proposition 14, we have

$$
\begin{aligned}
z & =[z \wedge(x \vee y)] \vee[z-(x \vee y)] \\
& \leq(x+y) \vee(x \wedge y \wedge z) \vee[z-(x \vee y)]=(x+y) \vee c
\end{aligned}
$$

and consequently,

$$
(x+y) \vee z \leq(x+y) \vee c,
$$

by $\left(l_{2}\right)$ and $\left(l_{8}\right)$. Now the second identity in (5.44) follows from relations (5.47) and (5.49).

Lemma 7. Let $(X, \wedge, \vee, \ominus, 0)$ be a B-ring. Then

$$
(x+y)+z=x+(y+z)
$$

for arbitrary $x, y, z \in X$.

Proof. By Proposition 20 and both identities in $7^{\circ}$ in Proposition 14, we have

$$
\begin{aligned}
(x+y)-z & =[(x-y) \vee(y-x)]-z \\
& =[(x-y)-z] \vee[(y-x)-z] \\
& =[x-(y \vee z)] \vee[y-(x \vee z)]
\end{aligned}
$$

and thus, by (5.41) and (5.42), we obtain

$$
\begin{aligned}
(x+y)+z= & {[(x+y)-z] \vee[z-(x+y)] } \\
= & {[x-(y \vee z)] \vee[y-(x \vee z)] } \\
& \vee[z-(x \vee y)] \vee(x \wedge y \wedge z)
\end{aligned}
$$

for arbitrary $x, y, z \in X$. 
Replacing $x$ by $y, y$ by $z$ and $z$ by $x$ in (5.51), we get

$$
\begin{aligned}
(y+z)+x=[y-(z \vee x)] \vee[z-(y \vee x)] & \vee[x-(y \vee z)] \\
& \vee(y \wedge z \wedge x)
\end{aligned}
$$

We see that the right hand sides of (5.51) and (5.52) coincide due to $\left(l_{3}\right)-\left(l_{5}\right)$, and hence (5.50) is true for all $x, y, z \in X$.

We will prove the following theorem:

Theorem 4. If $(X, \wedge, \vee, \ominus, 0)$ is a B-ring, then $(X,+, \cdot)$ is a Boolean ring with the operations + and $\cdot$ given by (5.39) and (5.40), i.e.

$$
x+y:=(x \vee y) \ominus(x \wedge y)=(x \vee y)-(x \wedge y)
$$

and

$$
x \cdot y:=x \wedge y .
$$

for all $x, y \in X$ and the neutral element of the operation + in $(X,+, \cdot)$ coincides with the above 0 , the order zero in $(X, \wedge, \vee, \ominus, 0)$.

Proof. Let $(X, \wedge, \vee, \ominus, 0)$ be a given $B$-ring. By (5.41) and $\left(l_{3}\right)$, we have $x+y=$ $y+x$ for $x, y \in X$, i.e. the operation + is commutative and its associativity was proved in Lemma 7.

In view of (5.39) and (l0), we get

$$
x+0=(x \vee 0) \ominus(x \wedge 0)=x \ominus 0=x \quad \text { for } x \in X,
$$

by the first equality in (3.10) in Proposition 13. Hence, the element $0=\inf X$ from the given $B$-ring is the neutral element (the unique 0 satisfying $x+0=x$ for all $x \in X$ ) of the operation + of addition. By equality (5.41) in Proposition 20 and by the second equality in (3.10) in Proposition 13, we have

$$
x+x=(x-x) \vee(x-x)=0 \vee 0=0 \quad \text { for } x \in X,
$$

due to $(l 0)$ or $\left(l_{2}\right)$, i.e. every element $x$ is the inverse to itself, with respect to the operation + of addition in $(X,+, \cdot)$.

In view of $\left(l_{3}\right)$, we have

$$
x \cdot y=x \wedge y=y \wedge x=y \cdot x \quad \text { for } x, y \in X,
$$

i.e. the multiplication $\cdot$ is commutative in $(X,+, \cdot)$. We have

$$
x \cdot x=x \wedge x=x \quad \text { for } x \in X,
$$

by $\left(l_{2}\right)$, so the multiplication $\cdot$ is idempotent in $(X,+, \cdot)$. Moreover, by $\left(l_{4}\right)$, we get

$$
(x \cdot y) \cdot z=(x \wedge y) \wedge z=x \wedge(y \wedge z)=x \cdot(y \cdot z) \quad \text { for } x, y, z \in X
$$


which proves that the multiplication $\cdot$ is associative in $(X,+, \cdot)$.

In view of Proposition 20, part $8^{\circ}$ of Proposition 14 and $(D)$, we get

$$
\begin{aligned}
x \cdot z+y \cdot z & =[(x \wedge z)-(y \wedge z)] \vee[(y \wedge z)-(x \wedge z)] \\
& =[(x-y) \wedge z] \vee[(y-x) \wedge z] \\
& =[(x-y) \vee(y-x)] \wedge z=(x+y) \cdot z
\end{aligned}
$$

for arbitrary $x, y, z \in X$. The last equality implies that the operation + of addition is distributive with respect to the operation - of multiplication.

The proof is completed.

The following assertion follows directly from Theorems 3 and 4 .

Theorem 5. If $(X, \vee, \backslash, 0)$ is a $\Delta$-join-semilattice, then $(X,+, \cdot)$ is a Boolean ring with + and $\cdot$ given by

$$
x+y:=(x \vee y) \backslash[x \backslash(x \backslash y)] \text { and } x \cdot y:=x \backslash(x \backslash y)
$$

for all $x, y \in X$ and the neutral element of the operation + in $(X,+, \cdot)$ coincides with the above 0 , the order zero in $(X, \vee, \backslash, 0)$.

Now assume that $(X,+, \cdot)$ is a Boolean ring (with the neutral element 0 of the operation + of addition) and define in $X$ the relation $\leq$ in the following way:

$$
x \leq y \quad: \Leftrightarrow x \cdot y=x
$$

We will prove that $(X, \leq)$ is a poset, satisfying conditions $(L)$ and $(L 0)$, i.e. a 0-lattice with the lattice operations $\wedge$ and $\vee$ defined as in Definition 3:

$$
x \wedge y:=\inf \{x, y\} \text { and } x \vee y:=\sup \{x, y\} \quad \text { for } \quad x, y \in X,
$$

with

$$
0=\inf X
$$

where 0 is the mentioned above neutral element of the operation + in the Boolean ring $(X,+, \cdot)$. Moreover, we will show that this 0-lattice is distributive and satisfies condition $(R)$, i.e. it is a $B$-ring, with the partial binary operation $\ominus$ of proper difference introduced according to Definition 7 , and the identities

$$
x \wedge y=x \cdot y \quad \text { and } \quad x \vee y=x+y+x \cdot y
$$

are satisfied for all $x, y \in X$.

We recall certain algebraic properties of groups and Boolean rings that we need in the proof of Theorem 6 .

Lemma 8. If $(X,+)$ is a group, then $x+y=x$ implies $y=0$ for any $x, y \in X$. If $(X,+, \cdot)$ is a Boolean ring, then it is commutative and $x+x=0$ for every $x \in X$. 
Proof. In a given group $(X,+)$, fix elements $x$ and $y$ such that $x+y=x$. Then

$$
y=0+y=((-x)+x)+y=(-x)+(x+y)=(-x)+x=0,
$$

which proves the first part of the lemma.

Since in a given Boolean ring $(X,+, \cdot)$ the multiplication is idempotent, we have

$$
x+y=(x+y)^{2}=x^{2}+y \cdot x+x \cdot y+y^{2}=x+y+y \cdot x+x \cdot y
$$

for arbitrary $x, y \in X$ and hence, by the first part of the lemma,

$$
y \cdot x+x \cdot y=0 \quad \text { for } x, y \in X
$$

Putting $y=x$ in (5.57), we get $0=x^{2}+x^{2}=x+x$ for each $x \in X$, which means that the last part of the lemma is true. Hence, by (5.57),

$$
y \cdot x=-x \cdot y=x \cdot y \quad \text { for } x, y \in X,
$$

which shows that the ring $(X,+, \cdot)$ is commutative, as stated in the middle part of the lemma. Thus the proof is completed.

Theorem 6. If $(X,+, \cdot)$ is a Boolean ring, then $(X, \wedge, \vee, \ominus, 0)$ is a B-ring in a poset $(X, \leq)$, where $\leq$ is defined by (5.53), the lattice operations $\wedge$ and $\vee$ are given by (5.54), the order zero 0 coincides with the neutral element of the addition in $(X,+, \cdot)$ and the operation $\ominus$, determined by condition $(R)$, is given by

$$
x \ominus y:=x+y \quad \text { for } x, y \in X \quad \text { suchthat } y \leq x .
$$

Moreover, the lattice operations $\wedge$ and $\vee$ defined in (5.54) are connected with the ring operations + and $\cdot$ in the given $(X,+, \cdot)$ by means of formulae in (5.56).

Proof. Let $(X,+, \cdot)$ be a Boolean ring. We start with proving that the relation $\leq$ defined in $(5.53)$ is a partial order, i.e. $(X, \leq)$ is a poset. In the proof of this fact as well as of properties of the operations + and - defined by (5.54) we will often apply the definition of $\leq$ given in (5.53).

By the assumption, we have $x^{2}=x$ and so $x \leq x$ for any $x \in X$, in view of (5.53). If $x \leq y$ and $y \leq x$, then $x \cdot y=x$ and $y \cdot x=y$, according to (5.53), so $x=y$ for any $x, y \in X$. Assume that $x \leq y$ and $y \leq z$ for given $x, y, z \in X$. By (5.53), we have $x \cdot y=x$ and $y \cdot z=y$, so

$$
x \cdot z=(x \cdot y) \cdot z=x \cdot(y \cdot z)=x \cdot y=x,
$$

which means that $x \leq z$, in view of (5.53) again. Consequently, $(X, \leq)$ is a poset.

If 0 denotes the neutral element of the addition + in the given Boolean ring, then $0 \cdot x=0$ and so, by (5.53), we have $0 \leq x$ for any $x \in X$. On the other hand, fix $a \in X$ such that $a \leq x$ and so $a \cdot x=a$ for all $x \in X$, due to (5.53). In particular, we have $a=a \cdot 0=0$. Hence 0 is the greatest lower bound of $X$ and equality (5.55) holds. 
Fix arbitrarily $x, y \in X$ and denote

$$
u:=x+y+x \cdot y \quad \text { and } \quad v:=x \cdot y .
$$

By associativity, commutativity and idempotency of the operation · of multiplication, we get

$$
v \cdot x=(x \cdot y) \cdot x=x^{2} \cdot y=x \cdot y=v \quad \text { and } \quad v \cdot y=x \cdot y^{2}=x \cdot y=v,
$$

i.e. $v \leq x$ and $v \leq y$, according to (5.53). But if $a \leq x$ and $a \leq y$, i.e. $a \cdot x=a$ and $a \cdot y=a$, then we obtain

$$
a \cdot v=a^{2} \cdot(x \cdot y)=(a \cdot x) \cdot(a \cdot y)=a \cdot a=a,
$$

due to the mentioned properties again, which means that $a \leq v$, according to (5.53). This shows that $\inf \{x, y\}=v \in X$.

Now, applying distributivity of + with respect to $\cdot$ and other properties of these operations, we obtain

$$
x \cdot u=x^{2}+x \cdot y+x^{2} \cdot y=x+x \cdot y+x \cdot y=x
$$

and

$$
y \cdot u=x \cdot y+y^{2}+x \cdot y^{2}=y+x \cdot y+x \cdot y=y,
$$

in view of Lemma 8 . Hence, by (5.53), we have

$$
x \leq u \quad \text { and } \quad y \leq u .
$$

On the other hand, if $x \leq a$ and $y \leq a$, then $x \cdot a=x$ and $y \cdot a=y$, so

$$
a \cdot u=a \cdot x+a \cdot y+(a \cdot x) \cdot y=x+y+x \cdot y=u,
$$

i.e. $u \leq a$, again due to (5.53). This and (5.59) mean that $\sup \{x, y\}=u \in X$.

We have thus shown that $\inf \{x, y\}$ and $\sup \{x, y\}$ exist in $X$ and moreover, according to the notation introduced in (5.54) and (5.58), the equalities in (5.56) hold for all $x, y \in X$. Consequently, the poset $(X, \leq)$ is a 0-lattice with the lattice operations $\wedge$ and $\vee$, given by (5.54) and (5.56), and the order zero equal to the neutral element 0 of the operation + of additivity in $(X,+, \cdot)$. We may denote it now by $(X, \wedge, \vee, 0)$. In view of (5.56) and properties of the operations + and $\cdot$ in $(X,+, \cdot)$, we have

$$
\begin{aligned}
x \wedge(y \vee z) & =x \cdot(y+z+y \cdot z)=x \cdot y+x \cdot z+x \cdot y \cdot z \\
& =x \cdot y+x \cdot z+(x \cdot y) \cdot(x \cdot z)=(x \wedge y) \vee(x \wedge z)
\end{aligned}
$$

for arbitrary $x, y, z \in X$, which proves that the 0-lattice $(X, \wedge, \vee, 0)$ is distributive.

Fix now elements $x, y \in X$ such that $y \leq x$ and put $z:=x+y$. By (5.56), Lemma 8 and properties of the operations + and $\cdot$ in $(X,+, \cdot)$, we get

$$
z \wedge y=z \cdot y=(x+y) \cdot y=x \cdot y+y^{2}=y+y=0
$$


and

$$
\begin{aligned}
z \vee y & =(x+y)+y+(x+y) \cdot y=x+(y+y)+x \cdot y+y^{2} \\
& =x+(y+y)+(y+y)=x,
\end{aligned}
$$

which means that $z$ satisfies the equalities in condition $(R)$. Consequently the 0 lattice $(X, \wedge, \vee, 0)$ is a $B$-ring in which the operation $\ominus$ is defined by $x \ominus y=z$, where $z:=x+y$ for any $x, y \in X$ such that $y \leq x$.

Theorems 3 and 6 imply the following assertion:

Theorem 7. If $(X,+, \cdot)$ is a Boolean ring, then $(X, \vee, \backslash, 0)$ is a $\Delta$-join-semilattice in a poset $(X, \leq)$, where $\leq$ is defined by (5.53), the operation $\vee$ is given by

$$
x \vee y:=x+y+x \cdot y \quad \text { for } x, y \in X,
$$

the order zero 0 coincides with the neutral element of the addition in $(X,+, \cdot)$ and the binary operation $\backslash$, determined by conditions $(\Delta)$ and $\left(I_{m}\right)$, is given by

$$
x \backslash y:=x+x \cdot y \quad \text { for } x, y \in X .
$$

Remark 8. Notice that in Theorems 4 and 5 we do not assume that the order unit $1=\sup X$ exists in $(X, \leq)$; consequently, the algebraic ring $(X,+, \cdot)$ in the assertions may not contain a unit, a neutral element of the operation - of multiplication. Similarly, in Theorems 6 and 7 we do not assume that the algebraic ring $(X,+, \cdot)$ contains a neutral element of the multiplication - and, consequently, the order unit $\sup X$ may not exist in $(X, \leq)$. Adding the respective assumptions we get the respective particular cases of the above theorems.

Acknowledgement. We express our thanks to Professor A. Kamiński for his inspiration and advices and to the referees for very valuable suggestions which allowed us to improve the text of our paper.

\section{References}

[1] B. Aniszczyk, J. Burzyk, A. Kamiński, Borel and monotone hierarchies and extensions of Rényi probability spaces, Colloq. Math. 51 (1987), 9-25.

[2] L. Beran, Orthomodular Lattices, Algebraic Approach, Academia/Reidel, Praque/Dordrecht 1984.

[3] G. Birkhoff, Lattice Theory, Amer. Math. Soc., Providence, Rhode Island, First edition 1940; Second revised edition 1961; Third edition 1967; Corrected reprint of the third edition 1979. 
[4] I. Chajda, Pseudosemirings induced by ortholattices, Czechoslovak Math. J. 46 (1996), 405-411.

[5] I. Chajda, G. Eigenthaler, A note on orthopseudorings and Boolean quasirings, Österreich. Akad. Wiss. Math.-Natur. Kl. Sitzungsber. II 207 (1998), 83-94.

[6] I. Chajda, H. Länger, Ring-like operations in pseudocomplemented semilattices, Discuss. Math. Gen. Algebra Appl. 20 (2000), 87-95.

[7] I. Chajda, H. Länger, M. Mączyński, Ring like structures corresponding to generalized orthomodular lattices, Math. Slovaca 54 (2004), 143-150.

[8] F. Chovanec, F. Kôpka, D-posets, In: K. Engesser, D. M. Gabbay, D. Lehmann (eds.), Handbook of Quantum Logic and Quantum Structures: Quantum Structures, 367-428, Elsevier, Amstredam, 2007.

[9] A. Dadej, K. Halik, Properties of differences in B-rings, J. Math. Appl. 34 (2011), 5-13.

[10] D. Dorninger, H. Länger, M. Mączyński, The logic induced by a system of homomorphisms and its various algebraic characterizations, Demonstratio Math. 30 (1997), 215-232.

[11] D. Dorninger, H. Länger, M. Mączyński, On ring-like structures occurring in axiomatic quantum mechanics, Österreich. Akad. Wiss. Math.-Natur. Kl. Sitzungsber. II 206 (1997), 279-289.

[12] D. Dorninger, H. Länger, M. Mączyński, On ring-like structures induced by Mackey's probability function, Rep. Math. Phys. 43 (1999), 499-515.

[13] D. Dorninger, H. Länger, M. Mączyński, Lattice properties of ring-like quantum logics, Internat. J. Theoret. Phys. 39 (2000), 1015-1026.

[14] D. Dorninger, H. Länger, M. Mạczyński, Concepts of measures on ring-like quantum logics, Rep. Math. Phys. 47 (2001), 167-176.

[15] D. Dorninger, H. Länger, M. Mạczyński, Ring-like structures with unique symmetric difference related to quantum logic, Discuss. Math. Gen. Algebra Appl. 21 (2001), 239-253.

[16] R. Frič, Remarks on statistical maps and fuzzy (operational) random variables, Tatra Mt. Math. Publ. 30 (2005), 21-34.

[17] R. Frič, Statistical maps: a categorical approach, Math. Slovaca 57 (2007), 41-57.

[18] R. Frič, M. Papčo, On probability domains, Int. J. Theor. Phys. 49 (2010), 30923100 .

[19] R. Frič, M. Papčo, On probability domains II, Int. J. Theor. Phys. 50 (2011), $3778-3786$. 
[20] R. Frič, M. Papčo, A categorical approach to probability, Stud. Log. 94 (2010), 215-230.

[21] G. Grätzer, General Lattice Theory, Birkhäuser Verlag, Basel-Boston-Berlin, First edition 1978; Second edition 1998; Reprint of the second edition 2003.

[22] K. Halik, A. Pydo, A note on Sierpiński-Pettis theorems in terms of generalized Boolean $\sigma$-algebras, Scientific Bulletin of Chełm 2 (2006), 67-72.

[23] M. F. Janowitz, A note on generalized orthomodular lattices, J. Natur. Sci. Math. 8 (1968), 89-94.

[24] G. Kalmbach, Orthomodular Lattices, Academic Press, London 1983.

[25] A. Kamiński, Distributors and Rényi's theory of conditional probabilities, in: Generalized Functions and Operational Calculus, Proc. Conf., Varna 1975, Publishing House of the Bulg. Acad. of Sciences, Sofia 1975, 111-121.

[26] A. Kamiński, On the Rényi theory of conditional probabilities, Studia Math. 79 (1984), 151-191.

[27] A. Kamiński, On extensions of Rényi conditional probability spaces, Colloq. Math. 49 (1985), 267-294.

[28] A. Kamiński, Generated $\sigma$-rings and $\sigma$-algebras, Institute of Mathematics Polish Academy of Sciences, Preprint 385, Waraszawa 1987, 1-36.

[29] A. Kamiński, Convolution of distributions and Rényi's theory of conditional probabilities, Integral Transforms and Spec. Funct. 6 (1998), 215-222.

[30] F. Kôpka, D-posets of fuzzy sets, Tatra Mt. Math. Publ. 1 (1992), 83-87.

[31] F. Kôpka, Quasi product on Boolean D-posets, Int. J. Theor. Phys. 47 (2008), 1625-1632.

[32] F. Kôpka, F. Chovanec, D-posets, Math. Slovaca 15 (1994), 21-34.

[33] M. Papčo, On measurable spaces and measurable maps, Tatra Mt. Math. Publ. 28 (2004), 125-140.

[34] M. Papčo, On fuzzy random variables: examples and generalizations, Tatra Mt. Math. Publ. 30 (2005), 175-185.

[35] M. Papčo, On effect algebras, Soft Comput. 12 (2008), 373-379.

[36] B. J. Pettis, On some theorems of Sierpiński on subalgebras of Boolean $\sigma$-rings, Bull. Acad. Polon. Sci. 19 (1971), 563-568.

[37] A. Rényi, On a new axiomatic theory of probability, Acta Math. Acad. Sci. Hungar. 6 (1955), 285-335. 
[38] A. Rényi, Probability Theory, Akadémiai Kiadó, Budapest 1970.

[39] W. Sierpiński, Une théorème générale sur les familles d'ensemble, Fund. Math. 12 (1928), 206-211.

[40] R. Sikorski, Boolean Algebras, Springer-Verlag, Berlin, Göttingen, Heidelberg, New York, First edition 1960; Second edition 1964.

[41] M. H. Stone, Postulates for Boolean Algebras and Generalized Boolean Algebras, Amer. J. Math. 57 (1935), 703-732.

[42] M. H. Stone, The theory of representations for Boolean algebras, Trans. Amer. Math. Soc. 40 (1936), 37-111.

[43] M. H. Stone, Algebraic characterizations of special Boolean rings, Fund. Math. 29 (1937), 223-303.

[44] T. Traczyk, Wstęp do teorii algebr Boole'a, PWN, Warszawa 1970.

[45] D. A. Vladimirov, Boolean Algebras, Nauka, Moscow 1969 (in Russian).

DOI: $10.7862 /$ rf.2013.4

\section{Aneta Dadej - corresponding author} email: khalik@gazeta.pl

Katarzyna Halik

email: apydo@wp.pl,

Institute of Mathematics,

University of Rzeszów,

ul. Rejtana 16A,

35-310 Rzeszów, Poland

Received 12.03.2013, Revisted 25.08.2013, Accepted 25.10.2013 